\title{
A Face Detection Method Used for Color Images
}

\author{
Wen-cheng Wang \\ Department of Information and Control, Weifang University, Weifang 261061, \\ China \\ wwchpaper@126.com
}

\begin{abstract}
To the problem of face detection in color images, a novel method is proposed based on skin color segmentation and geometry features. Firstly, some common color models are analyzed, and a large amount of skin images are used to establish an $\mathrm{YCbCr}$ color model for region segmentation. Then, the morphological processing is executed on the binary image, and the facial regions filtering is conducted by adopting some geometry constraints such as Euler number, the ratio of width and height, centroid. Finally, the face region is located and labeled with a rectangle. Experimental results showed that the proposed method can equilibrate the unbalance between good accuracy and real-time performance to some extent, and is effective in face location.
\end{abstract}

Keywords: Face Detection, Color Space, Color Image, Skin Color Model

\section{Introduction}

Face detection is a key link in automatic face recognition system and a popular hotspot in computer vision and pattern recognition research, which has been widely applied in content-based image retrieval, access control system, video surveillance, video conferencing and intelligent man-machine interaction and other fields $[1,2]$. For face detection, after years of research and development, a large number of classic algorithms were emerged, such as template matching algorithm [3], heuristic model-based algorithm [4], statistical model-based algorithm [5], etc., Among these approaches, template matching approach describes overall facial features or several separate facial features through extracting several standard face pattern. It has good results for face extraction with the simple background, but to complex background, the retrieving time is too long. Heuristic model-based approach, through analyzing prior knowledge of face, extracts the distribution rules of face structure to describe the geometric relationship between the facial features. It has better detection result for the frontal face against the simple background, but for the deflecting face, it shows its limits; Statistical model-based approach, for example, the ANN (Artificial Neural Network) approach and SVM (Support Vector Machine) approach, have high accuracy in detection, but these require a lot of faces and non-face training samples which need a long time for training. Furthermore, for face detection in color images, these approaches are only suitable for the detection of the frontal face, and it costs a lot of time. For the color face image, skin color is an effective feature. Compared with other geometric features, the skin color can be distinguished from colors of most background objects, and will not be impacted by the changes of shape, size, rotation, and expression [6].

Therefore, the main work of this paper, on the basis of analyzing four kinds of color space: RGB, HIS, YUV, and YCbCr, is to study the clustering features of the skin color in different color spaces, establish a practical and reliable color mixing model, and finally, use $\mathrm{YCbCr}$ color space for skin color detection, screen the 
candidate regions through the integration of the geometric characteristics of the face, further remove the similar skin region and other regions of body exposed, and continue to narrow search scope. The experiment shows that such integrated algorithm has certain advantages and robustness in face detection in color image.

\section{Color Space Theory}

Before skin color segmentation, the selection of the color space and the establishment of the skin color model are mainly involved [7-11]. The color spaces which are often used for skin color analysis are as follows.

(1) RGB color space. RGB color space is one of the most common color space representation approaches. It generates various colors by adjusting the red, green and blue values, which applies to computer storage and display. As the three primary colors $(R, G, B)$ contain both Intensity and color information, they have a high correlation.

(2) HIS color space. HIS color space is also called as hue, saturation, and intensity model. This color space reflects a way used by human in observation of color, and meanwhile, it is conducive to the image processing. In the use of color information, the advantage of this format lies in that it separates $I$ (Intensity) and two parameters that reflect the essential characteristics of color, i.e., $H$ (Hue) and $S$ (Saturation). The conversion relationship between RGB color space and HIS respective components is as follows:

$$
\left\{\begin{array}{l}
I=\frac{R+G+B}{3} \\
S=1-\frac{3}{(R+G+B)}[\min (R, G, B)] \\
H=\arccos \frac{\frac{1}{2}[(R-G)+(R-B)]}{\sqrt{(R-G)^{2}-(R-B)(G-B)}}
\end{array}\right.
$$

(3) YUV color space. YUV color system is used in Europe system (belong to PAL system), and the intensity information is often called as component $Y$, color difference signals is called as component $U$ and component $V$, which are used to describe the amount of the red and blue in the image. The combination of these signals is referred to as YUV color space, and the red, green, and blue together is the brightness of the image. The formula for the conversion from RGB to YUV space is as follows:

$$
\begin{aligned}
& Y \\
& U \\
& V
\end{aligned}\left[\begin{array}{ccc}
0.299 & 0.587 & 0.114 \\
-0.148 & -0.289 & -0.437 \\
0.615 & -0.515 & -0.100
\end{array}\right]\left[\begin{array}{l}
R \\
G \\
B
\end{array}\right]
$$

(4) $\mathrm{YC}_{\mathrm{b}} \mathrm{C}_{\mathrm{r}}$ color space. This color space is a color representation model with the studio quality as the objective and that is adopted in CCIR601 encoding scheme. It is widely used in color display of the television and other fields. It also has advantages in the separation of the intensity component in the HSV format. Since it can be obtained from the linear change of RGB format, it can be directly applied to the clustering analysis of the object color. The formula for the conversion from $\mathrm{RGB}$ to $\mathrm{YC}_{\mathrm{b}} \mathrm{C}_{\mathrm{r}}$ space is as follows: 
(3)

$$
\left[\begin{array}{c}
Y \\
C b \\
C r \\
1
\end{array}\right]=\left[\begin{array}{cccc}
0.2990 & 0.5870 & 0.1140 & 0 \\
-0.1687 & -0.3313 & 0.5000 & 128 \\
0.5000 & -0.4187 & 0.0813 & 128 \\
0 & 0 & 0 & 1
\end{array}\right]\left[\begin{array}{c}
R \\
G \\
B \\
1
\end{array}\right]
$$

$Y$ represents the intensity of color, $C_{\mathrm{b}}$ represents the blue component, $C_{\mathrm{r}}$ represents the red component, $C_{\mathrm{r}}$ with $C_{\mathrm{b}}$ jointly represents the hue information, and $\mathrm{Cr}$ and $C_{\mathrm{b}}$ are two-dimensionally independent. Its main advantage is that it can effectively separate intensity and hue, and the hue information in many applications for color image segmentation is very effective.

\section{Establishment of Skin Color Model}

In the RGB space, three primary colors represent the color as well as brightness. Due to changes in ambient lighting, the brightness may make it complex to detect faces. So it does not have good clustering characteristics for the face skin, and is not suitable for skin models. Therefore, in skin color detection, it needs to transfer images from the RGB color space to another color space, making it easy to separate the skin color and the background color. According to studies, $\mathrm{YC}_{\mathrm{b}} \mathrm{C}_{\mathrm{r}}$ space has a good effect in distinguish skin color and background color, and the skin color shows a better clustering characteristics. Therefore, $\mathrm{YC}_{\mathrm{b}} \mathrm{C}_{\mathrm{r}}$ space is selected as the mapping space for skin distribution statistics in the experiment of this paper. The advantages of this space are that it is less affected by changes in brightness, and is two-dimensionally and independently distributed, which can preferably limit skin color distribution region. In order to make skin color distribution statistics more representative, 200 face colors of yellow race with different faces, under different lightings, of different ages, with different genders and different regions of the body (including the face, arm, legs and trunks) are selected as samples. The regions with high or low brightness are avoided to the greatest extent during sampling. Figure 1 shows skin color samples, with the size of $30 \times 30$ pixels for each image.

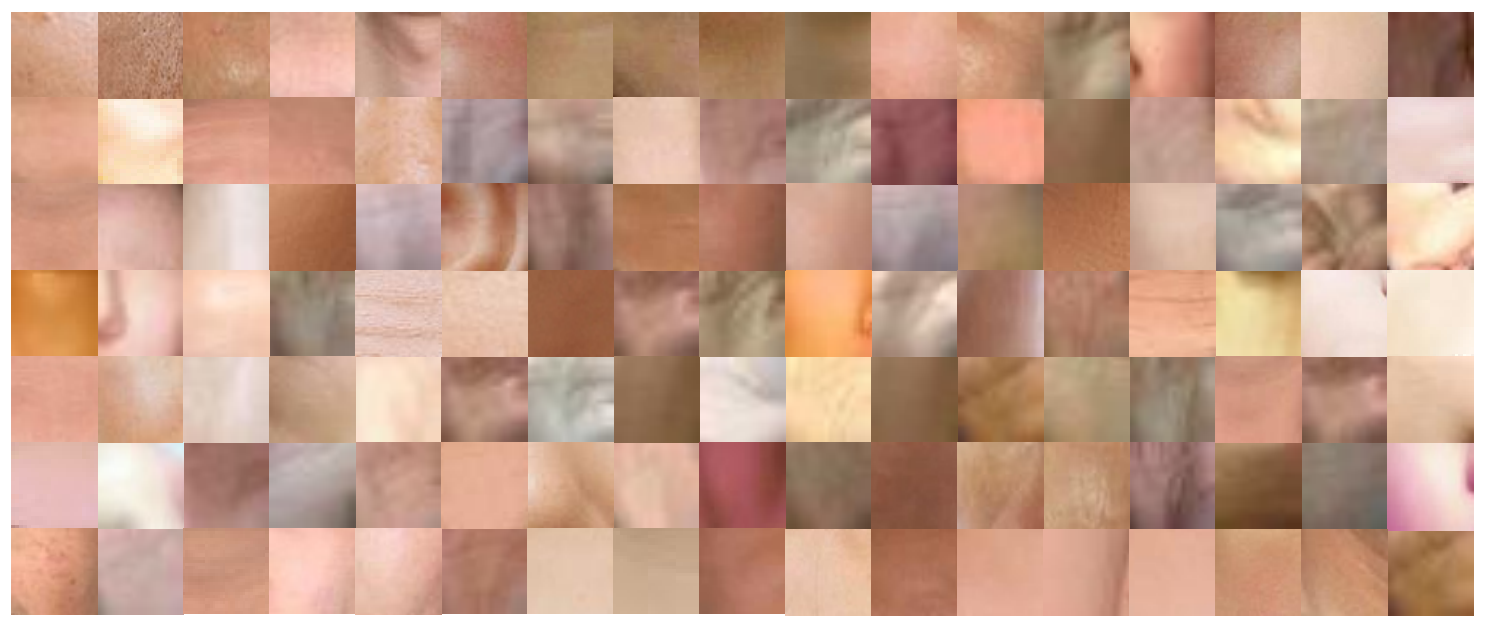

Figure 1. Sample Set of Skin Color

Through statistics it is proved that the skin color distinguishing between different ethnic groups is mainly affected by the brightness, but less affected by hue. In the experiment, face region below the eyes is selected as the statistical region of the skin color. Through statistical analysis of maps of these face color samples in the $C_{\mathrm{b}}$ and $C_{\mathrm{r}}$ spaces, we can know that in the $\mathrm{YC}_{\mathrm{b}} \mathrm{C}_{\mathrm{r}}$ space, the skin colors are within a stable range in 
the $C_{\mathrm{b}}$ and $C_{\mathrm{r}}$ information. A Gaussian model is shown in Figure 2. The histogram of the components of the skin color on $Y, C_{\mathrm{b}}$, and $C_{\mathrm{r}}$ space is shown in Figure 3.

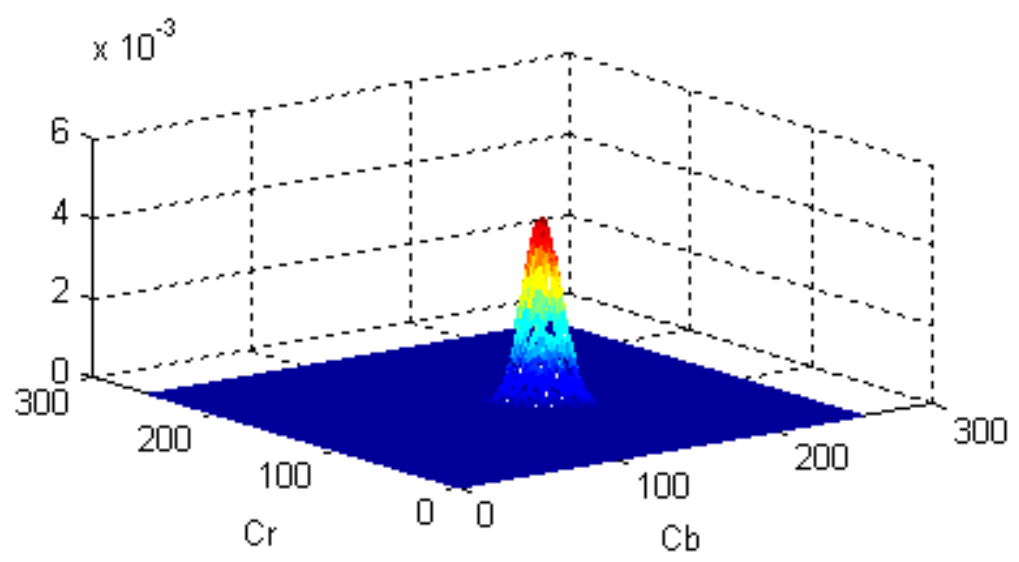

Figure 2. The Distribution Map of Gauss Model

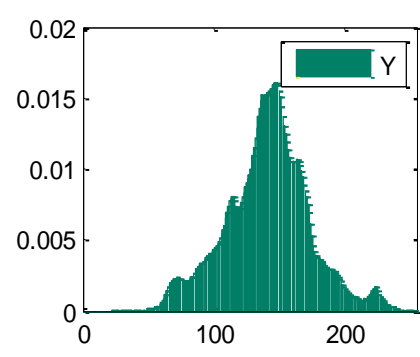

(a) Y component

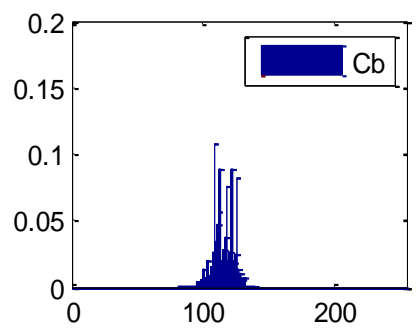

(b) $\mathrm{C}_{\mathrm{b}}$ component

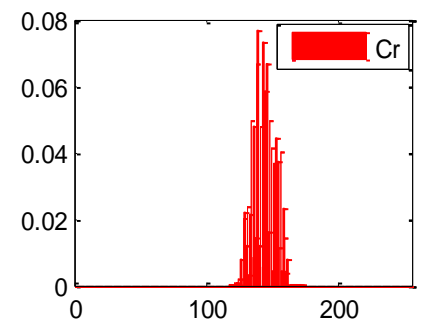

(c) $\mathrm{C}_{\mathrm{r}}$ component

Figure 3. The Histograms of Each Skin Color Component

Therefore, through skin color model, skin color similarity of a single pixel can be calculated to get a grayscale image segmentation using the above function image. If 1 is used to represent the skin region, and 0 represents a non-skin color region, the discriminant function of the skin color can be obtained, as shown in the formula (4) below.

$$
\left\{\begin{array}{lc}
1 & \left(100 \leq C_{b} \leq 128\right) \&\left(138 \leq C_{r} \leq 168\right) \\
0 & \text { otherwise }
\end{array}\right.
$$

The binary image obtained by the discriminant function is shown in Figure 4 below:

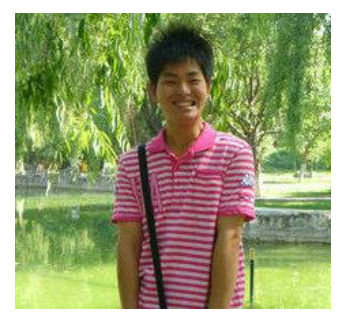

(a) Original image

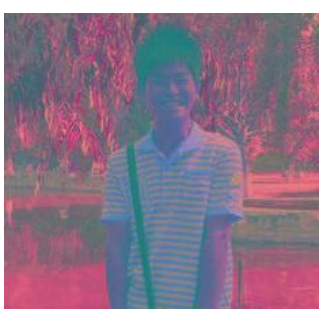

(b) $\mathrm{YC}_{\mathrm{b}} \mathrm{C}_{\mathrm{c}}$ color space

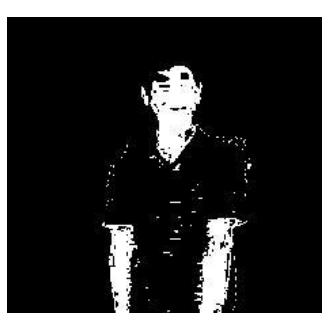

(c) Binary image

Figure 4. Skin Color Analysis and Image Segmentation

\section{Morphological Processing and Regions Screening}




\subsection{Morphological Processing}

As the skin region just processes $C_{\mathrm{b}}$ and $C_{\mathrm{r}}$ components of the $\mathrm{YC}_{\mathrm{b}} \mathrm{C}_{\mathrm{r}}$ space, the following situations are inevitable after the binary segmentation: one is the inclusion of noise, resulting in connecting the face region with the surrounding non-face region, or isolated points determined as skin; another one is that the local regions of the face such as the eyebrow, eyes and other non-skin regions cause the non-full filling of the face. For this, we apply morphological opening operator to remove these isolated background regions. It can remove small isolated points, burrs and bridges, while the total position remains unchanged. The dilation operation symbol is " $\oplus$ ", and the image combination $A$ uses the structural element $B$ for the dilation, expressed as $A \oplus B$, which is defined as:

$$
A \oplus B=\left\{x \mid(\hat{B})_{x} \cap A \neq \phi\right\}
$$

Erosion operation symbol is " $\Theta$ ", and the image combination $A$ uses the structural element $B$ for the erosion, expressed as $A \Theta B$, in the formula:

$$
A \Theta B=\left\{x \mid(\hat{B})_{x} \subseteq A\right\}
$$

The Formula (6) shows that the results by using $A$ with $B$ satisfy that after translating $B$ for $x, B$ is still fully contained in $x$ set of $A$.

In our application, conducting the opening operation for the overall detected skin can more effectively remove small isolated regions. After the above operation, the erosion operation is used to remove isolated noises, and the expansion operation is used to fill the non-skin region of face, so that the whole image will appear as a fully filled connected region. Therefore, the closing operation is "•", for which, expansion operation is firstly conducted and then the erosion operation is conducted. If $A$ is closed by $B$, it is expressed as $A \cdot B$, defined as follows:

$$
A \bullet B=(A \oplus B) \Theta B
$$

(7)

Assuming that the image is $P(x, y)$ after the color detection, and the image is $\operatorname{Seg}(x, y)$ after closed operation, then:

$$
\operatorname{Seg}(x, y)=P(x, y) \bullet B
$$

where, $B$ is the structure element.

\subsection{Regions Screening}

After processing with the filtering approach based on mathematical morphology and regional marking, small blocks of noise in the image are cleared, but since the background is very complex, influence factors are numerous, there may be bear arms or legs and other non-face regions are falsely detected as candidate face region. In order to try to delete the non-face images, they are mainly verified according to some prior knowledge of geometry, such as shape size, length-width ratio, the length-width shaft ratio of the approximate ellipse, pixels share, so as to exclude the non-face regions and retain face skin color blocks.

For face region, since the presence of non-color regions such as the eye, mouth, eyebrows, there will be one or more "holes" (non-face region) in the face region. You can get rid of those skin regions that do not contain holes based on such knowledge. For this, the calculation of the number of holes in the candidate face regions involves the Euler number. Euler number is defined as that the number of connected component minuses the number of the holes, as shown in the formula:

$$
E=C-H
$$


(9)

Where, $E, C$ and $H$ is respectively Euler number, connected component and hole number. It can be obtained from the above formula:

$$
H=C-E
$$

Taking into account the growing region for each skin color, $C=1$, so the $H=1-E$.

In the experiment, the Euler number of each block is calculated, which reflects the number of holes. Since the eyes, nose and lips on the face will appear in the black holes after the above steps, set a threshold with the calculated Euler number. When the block Euler number> 0, the block will be deemed as face region, and then conduct the selection of the candidate face region, and vice versa, it will be deemed as the non-face region.

Furthermore, the width-height ratio of the bounding rectangle of face also uses region screening approach:

$$
\text { ratio }=\frac{\operatorname{Re} c t_{-} w}{\operatorname{Re} c t_{-} h}
$$

Where the $\operatorname{Re} c t_{-} h$ is the width of the bounding rectangle, and the $\operatorname{Re} c t_{-} w$ is the height of the bounding rectangle. In this paper, the range of the ratio is $(0.5,2)$, and the candidate regions that do not belong to this range will be directly deleted. Figure 5 shows the image before and after the screening.

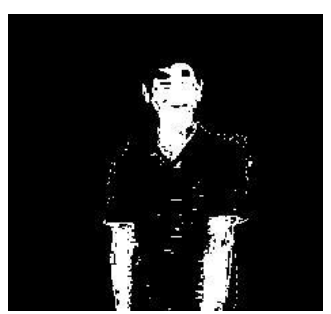

(a) Binary image

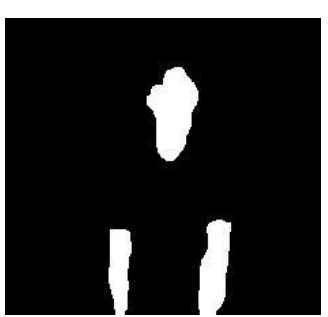

(b) Morphologic processing

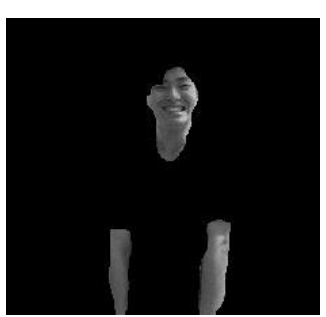

(c) Mask image

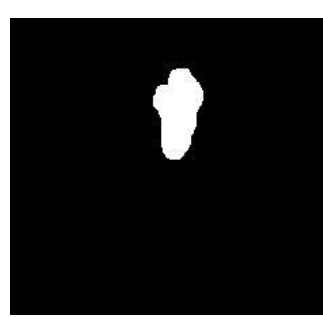

(d) Region screened

Figure 5. The Facial Region Selection

\section{Analysis of the Experimental Results}

In order to verify the effectiveness of the approach, we use Matlab to achieve the algorithm. The test of this paper is conducted on the self-established color face database. Some of the image in this database is downloaded from the Internet, and others come from the shooting by the laboratory digital camera. The complexity of the background of the image varies, and the illumination conditions are different, the faces in the image have different expressions and different poses, and each image is also different in size. Face detection and location process are shown in Figure 6. 


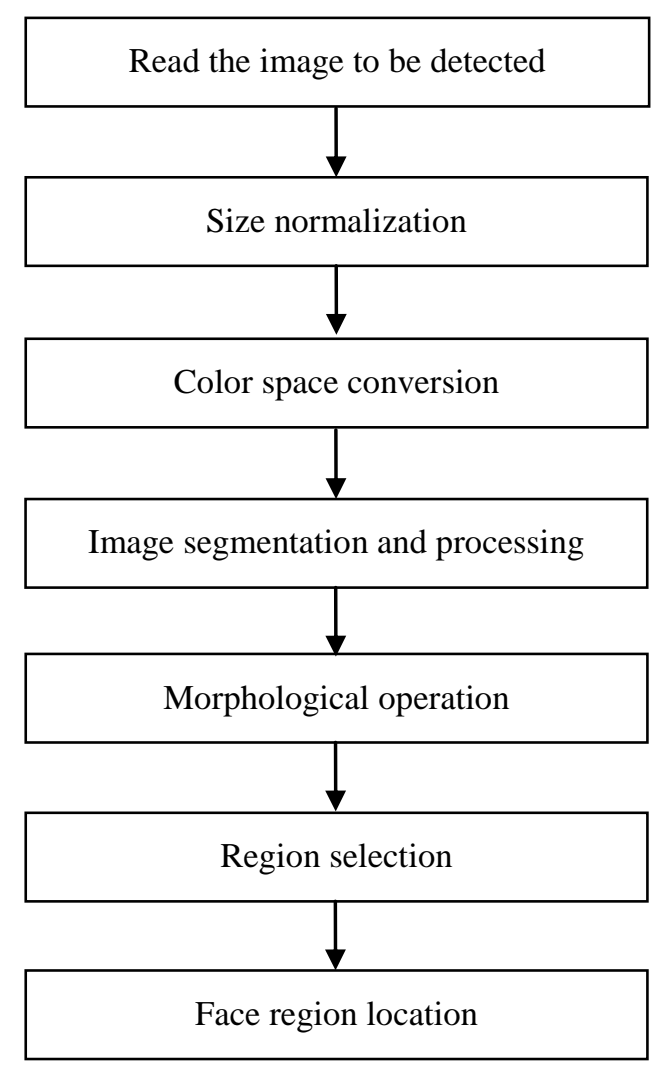

Figure 6. Flowchart of Face Detection

Some experimental examples are shown in Figure 7, in which, each face image is marked with red rectangle frame after location. The Figure 7 (a) shows the face detection with complex background, Figure 7 (b) is face detection and location with simple background, Figure 7 (c) is the location when the face deflects. From the test results, it shows that the skin color is important information of the face, which is independent of the details of the facial features and applicable to rotation, expression and other changes. It has relative stability and differs from color different from the color of most background objects.

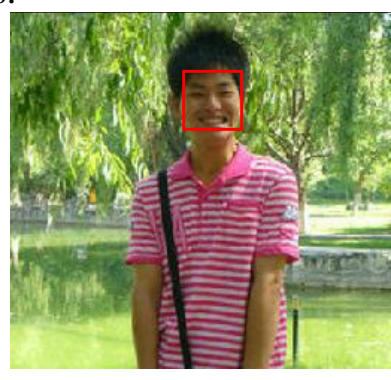

(a) Complex background

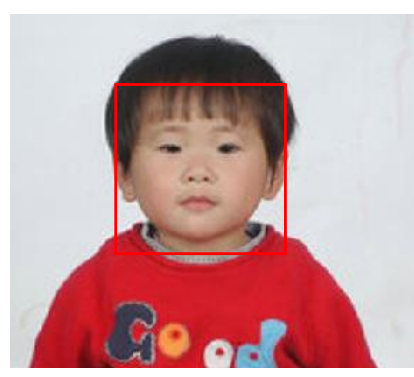

(b) Simple background

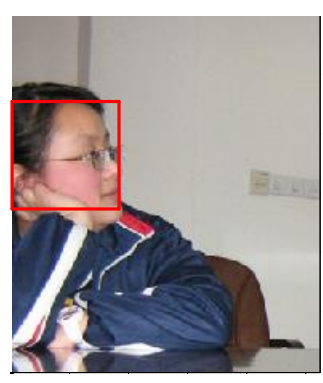

(c) Face deflected

Figure 7. Examples of Face Detection

For all samples used for the experiment, in order to summarize their overall effect, the equations for detection rate and accuracy are defined as follows:

$$
\alpha=\frac{N_{\alpha}}{N}
$$




$$
\beta=\frac{N_{\beta}}{N}
$$

$$
\gamma=\frac{N_{\gamma}}{N}
$$

where $\alpha$ is the accuracy of the skin color segmentation, $N_{\alpha}$ is the number of the faces accurately detected, $N$ is the total number of the faces, $\beta$ is the omission ratio of the skin color segmentation, $N_{\beta}$ is the number of the faces omitted, $\gamma$ is the false detection rate, and $N_{\gamma}$ is the number of the faces falsely detected

The final result is shown in Table 1.

Table 1. Comparison of Experimental Results

\begin{tabular}{cccc}
\hline Testing set & $\alpha$ & $\beta$ & $\gamma$ \\
\hline Single face image & 87.9 & 12.3 & 12.1 \\
Multiple faces image & 85.5 & 15.6 & 14.5 \\
\hline
\end{tabular}

The results in Table 1 shows that, under the normal lighting condition, this algorithm have good location effect for single face image and multiple-face image. Through analyzing the images, it is found that image color is severely distorted, the background region is similar to the skin color region, the hair covers eyes, and the face rotation angle is too large, all of which are the main reasons that cause the leak detection. Future work will focus on the further research in these regions, and meanwhile, conduct the research on the face detection with better integration of spatial information, face prior information and grey information.

\section{Conclusion}

This paper proposes an approach that realizes the face detection through the combination of skin color segmentation and geometric features. The algorithm uses color model of the $\mathrm{CbCr}$ space established by statistical approach to detect skin color regions, and divides the skin colors by the use of consistency of the face skin color in itself and the relative difference between the face and skin color background. The algorithm uses morphological operators in the divided regions for the expansion and erosion operation, which effectively removes some the noises. The region screening based prior knowledge screen the arms, clothing and other non-face regions, which facilitates the subsequent face location. The experiment shows that this can accurately extract the face region, and it is still effective for the image with the deflected face, and obtains good effect. It can be widely used for coarse location of the face in the color image, and has a strong adaptability.

\section{Acknowledgements}

This work has been supported by National Natural Science Foundation of China (Grant No. 61403283), Shandong Provincial Natural Science Foundation (No. ZR2013FQ036), the Spark Program of China (No. 2013XH06034), the Spark Program of Shandong Province (No. 2013XH06034) and Technology Development Plan of Weifang City(No. 201301015).

\section{References}


[1]. N. Sun, C. Zou and L. Zhao, "Face Detection: A Review”, Journal of Circuits and Systems", vol. 11, no. 6, (2006), pp.101-108.

[2]. S. Phung, A. Bouzerdoum and D. Chai, "A novel skin color model in YCbCr color space and its application to human face detection", Proceedings of International Conference on Image Processing, (2002), pp. 289-292.

[3]. A. Faizan, N. Aaima and A. Zeeshan, "Image-based Face Detection and Recognition: State of the Art", International Journal of Computer Science Issues, vol. 9, no. 1, (2012).

[4]. R. Brunelli and T. Poggio, "Face recognition: Features versus templates", IEEE Transactions on Pattern Analysis and Machine Intelligenee, vol. 15, no. 10, (1993), pp. 1042-1052.

[5]. R. L. Hsu, A. Mohamed and A. K. Jain, "Face detection in color images", IEEE Trans on Pattern Analysis and Machine Intelligence, vol. 24, no. 5, (2002), pp. 696-706.

[6]. H. Rowley, S. Baluja and T. Kanade, "Neural network-based face detection", IEEE Transactions on Pattern Analysis and Machine Intelligence, vol. 20, no. 1, (1998), pp. 23-38.

[7]. J. Cai and A. Goshtasby, "Detecting human faces in color images", Image and Vision Computing, vol. 18, no. 1, (1999), pp. 63-75.

[8]. W. Yun, D. Kim and H. Yoon, "Fast Group Verification Systemfor Intelligent Robot Service", IEEE Transactions on Consumer Electronics, vol.53, no.4, (2007), pp.1731-1735.

[9]. Z. Lei and S. Li. "Fast Multi-scale Local Phase Quantization Histogram for Face Recognition", Pattern Recognition Letters, vol. 33, no. 13, (2012), pp. 1761-1767.

[10].K. Ali, F. Fleuret, D. Hasler and P. Fua, "A real-time deformable detector", IEEE Transactions on Pattern Analysis and Machine Intelligence, vol. 34, no. 2, (2012), pp. 225-239.

[11].W. Wang, "Fast Face Detection Method Based on Skin Color", Journal of Convergence Information Technology, vol. 6, no. 9, (2011), pp.50-57.

\section{Author}

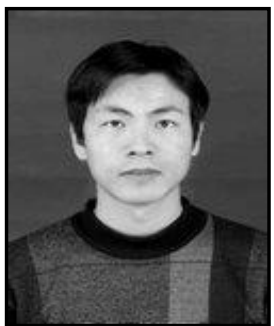

Wencheng Wang Received the Ph.D. degree in 2011. And now he is working in Weifang University. His group has published and authored more than 40 papers on academic journals and conferences, four book chapters and 7 patents. His main research interests include digital information processing and intelligent computing. 
International Journal of Signal Processing, Image Processing and Pattern Recognition Vol. 8, No. 2 (2015) 